\title{
Tetrahydrozoline Hydrochloride
}

National Cancer Institute

\section{Source}

National Cancer Institute. Tetrahydrozoline Hydrochloride. NCI Thesaurus. Code

C47749.

The hydrochloride salt form of tetrahydrozoline, an imidazole derivative with

sympathomimetic property. Applied locally to the eye or nose, tetrahydrozoline binds to

and activates alpha-adrenergic receptors, resulting in vasoconstriction and decreased nasal and ophthalmic congestion. 